\title{
Grommet Surgery in Children with Orofacial Clefts in England
}

\section{ABSTRACT}

Objective: To assess grommet insertion practice in the first five years of life among children with an orofacial cleft in England.

Design: Analysis of national administrative data of hospital admissions.

Setting: National Health Service hospitals, England.

Patients: Patients born alive between 1997 and 2005 who underwent surgical cleft repair. Intervention: Children receiving grommets before the age of five.

Outcome measures: The proportion of children receiving grommets before the age of five, the timing of the first grommet insertion and the proportion of children having repeat grommet insertions were examined according to cleft type, the absence or presence of additional anomalies, socio-economic deprivation, and region of residence.

Results: 8,269 children were included. Before the age of five, 3,015 (36.5\%) children received grommets. Of these, $33.2 \%$ received their first grommets at primary cleft repair and $33.3 \%$ underwent multiple grommet insertion procedures. The most common age for the first procedure was between six and 12 months. Children with a cleft affecting the palate were more likely to receive grommets than children with a cleft lip alone ( $45.5 \%$ vs. $4.5 \%)$. Grommet insertion practice also varied according to year of birth, absence or presence of additional anomalies, socio-economic deprivation, and region of residence.

Conclusion: Grommets practice in children with a cleft appears to vary according to their clinical characteristics. The differences in practice observed according to deprivation and region of residence need to be further explored.

KEYWORDS: Cleft lip, cleft palate, ventilation middle ear, grommet insertion, otological surgical procedure 


\section{INTRODUCTION}

Otitis media with effusion (OME) or 'glue ear' is a common childhood condition characterised by an accumulation of fluid within the middle ear space that can cause hearing impairment (National Institute for Health and Clinical Excellence, 2008). Children with cleft palate $(\mathrm{CP})$, with or without cleft lip $(\mathrm{CP} \pm \mathrm{L})$, are highly susceptible to OME because the palatal anomaly is associated with both structural and functional abnormality of the Eustachian tube leading to failure of middle ear ventilation (National Institute for Health and Clinical Excellence, 2008; Flynn et al., 2009). Among children with $\mathrm{CP} \pm \mathrm{L}, \mathrm{OME}$ presents at an early age, has a prolonged course and a high rate of recurrence (Atkinson, 2009).

OME may be managed conservatively with or without the use of hearing aids or surgically with the insertion of grommets (ventilation tubes). The National Institute for Health and Clinical Excellence (NICE) Guideline on 'Surgical management of OME in children' recommends that grommets should be offered as an alternative to hearing aids in children with $\mathrm{CP} \pm \mathrm{L}$ who have $\mathrm{OME}$ and persistent hearing loss, and that the insertion of grommets at primary repair of the CP should be performed only after careful otological and audiological assessment, rather than on a routine prophylactic basis (National Collaborating Centre for Women's and Children's Health, 2008; National Institute for Health and Clinical Excellence, 2008).

A systematic review of the management of $\mathrm{OME}$ in children with $\mathrm{CP} \pm \mathrm{L}$ found insufficient evidence to support the practice of early routine grommet insertion (Ponduri et al., 2009). The review included 18 small heterogeneous studies published between 1974 and 2004. The authors highlighted the need for further research to survey current protocols and practice for children with $\mathrm{CP} \pm \mathrm{L}$. National audits to provide information on varying management practices were also recommended by the NICE guidance. 
Identifying factors increasing the likelihood of a child with $\mathrm{CP} \pm \mathrm{L}$ receiving grommets is useful for those commissioning and planning cleft services and for counselling parents and carers. However, there is little information on grommet practice among children with $\mathrm{CP} \pm \mathrm{L}$ and the available data are limited to studies with small sample sizes. A recent systematic review (Kuo et al., 2014) of five small studies with a total of 384 patients across five countries (Japan, Hong Kong, UK, Ireland and New Zealand) examined grommet use for OME in children with CP. Kuo and colleagues estimated the frequency of grommet insertion, which ranged from $38 \%$ to $53 \%$ during a follow-up period of four to nine years. A much higher grommet insertion rate has been reported in the United States, where $98 \%$ of the 86 children with $\mathrm{CP} \pm \mathrm{L}$ received grommets in the first five years of life (Szabo et al., 2010).

Using national data on all hospital admissions in England, the aim of the present study was to examine grommet insertions among children with an orofacial cleft within the first five years of life. Our objectives were to examine on a national basis the proportion of children receiving grommets, the timing of the first grommet insertion, including whether this was at the time of primary cleft repair, and the proportion of children undergoing repeat insertions. For comparison, we also sought to calculate the corresponding background rates of grommet insertion among all live-born children in England across the same time period. 


\section{METHODS}

\section{Ethical considerations}

The study is exempt from UK National Research Ethics Committee approval as it involved analysis of an existing dataset of anonymised data for service evaluation. The Clinical Effectiveness Unit was given approval to use hospital episode statistics (HES) data for the evaluation of clinical practice and outcomes within NHS hospitals.

\section{Data source}

The HES database, with records on all admissions to NHS hospitals in England, provided records of hospital admissions up to 31 January 2011 (Hospital Episode Statistics, 2010).

\section{Patients}

As hospital admission records were available until January 2011 only, children born between 1 January 1997 and 31 December 2005 were identified to allow for a five-year follow-up period. Children were included if they had at least one HES record with a diagnosis code for cleft lip and/or cleft palate (CL/P) (ICD-10; Q35 (cleft palate), Q36 (cleft lip), Q37 (cleft palate with cleft lip) International Classification of Diseases 10th revision) as well as a procedure code for a primary cleft repair (OPCS-4; F031 (lip), F291 (palate) Classification of Surgical Operations and Procedures 4th Revision), not restricted to the five-year follow-up. Patients who were identified in HES as 'private' and whose postcode was 'unavailable/not applicable' ( $\mathrm{n}=97)$ were excluded from analyses, as their care may not reflect typical otological and audiological practice in England. 
Grommet Surgery

Children receiving grommets before the age of five years were identified by the presence of the grommet procedure code (OPCS-4; D151) in any of the procedure code fields within a HES record.

Follow-up

Data on hospital admissions were available up to January 2011. The follow-up period of five years was selected as the incidence of OME in childhood reduces with age (Møller, 1981) as does the proportion of those with hearing loss (Gould, 1990). It has been reported that very few children with a cleft require first-time grommets after five years (Kwan et al., 2011).

\section{Additional anomalies}

The presence of any one of 33 ICD-10 diagnostic codes (Appendix 1) representing congenital malformations and chromosomal abnormalities, in any of the diagnosis code fields of a HES record was used to identify children with additional congenital anomalies. Data for these children were analysed separately, as the presence of these diagnoses may have a major impact on their treatment. 
Cleft type

Orofacial clefts were grouped as cleft lip only (CL), cleft palate only (CP), unilateral cleft lip and palate (UCLP) or bilateral cleft lip and palate (BCLP) according to the presence of selected procedure codes (OPCS 4) and/or diagnosis codes (ICD-10) in any of the available HES records. A stepwise, hierarchical approach was employed. First, the cleft repair procedure codes (F03, F29, F30, F32) were used to identify CL, CP, Cleft lip and palate (CLP). Second, the CLP group was separated in to UCLP and BCLP using the diagnosis code.

\section{Socio-economic deprivation}

The Index of Multiple Deprivation (IMD) is a composite measure of deprivation reflecting a range of social and economic dimensions (Office of the Deputy Prime Minister, 2004). IMD ranks 32,482 small areas in England, each of which covers an average population of around 1,500 people or 400 households. Deprivation scores were allocated to each record based on the patient's postcode of residence. We grouped the patients into five socioeconomic categories based on fifths of the national ranking of these areas. We sought to determine whether grommet practice varied across these quintiles of IMD.

\section{Region of residence}

There are nine regional cleft networks in England. Grommet surgery was examined according to the regional cleft network in which the patient resided at the first primary repair record to determine whether there was a variation in practice between cleft networks. 


\section{Background rate of grommet insertions}

To put into context the grommet insertion rates among children with a cleft, we calculated the background rate of grommet insertions and the rate of multiple insertions among all live-born children (including those with a cleft and other medical diagnoses) in England across the same time period (1997-2005 births). Grommet insertions in the first five years of life were identified through HES using the same methods as described above. The number of live births in England across the study period was calculated using information from the Office for National Statistics (2008) and the Welsh Government (2015). The background grommet rates were also calculated for those without a cleft.

\section{Analyses}

The HES data underwent validation checks and duplicate records were removed. The proportion of children receiving grommets at their primary cleft repair, having at least one admission for grommet insertion and the proportion having repeat procedures within the first five years of life were examined. The age at which the procedure was first performed was analysed using Kaplan-Meier estimates (Kaplan and Meier, 1958). Data were analysed according to the absence or presence of additional medical anomalies, cleft type classification, deprivation quintile and region of residence. Trends across birth years and deprivation quintiles were examined for statistical significance using a nonparametric test for trend across ordered groups (Cuzick, 1985). The $\chi^{2}$ test was used to assess variations in proportions across non-ordered groups, such as cleft type classification. A p value $<0.05$ was 
considered statistically significant. All statistical calculations were performed in Stata 11 (Statacorp, College Station, TX, USA).

\section{RESULTS}

\section{All cleft children}

8,269 children with CL/P were identified. A total of 4,306 grommet insertion procedures were performed among 3,015 (36.5\%) children before the age of five years (Table 1). Among these children, 1,001 (12.1\% of all children and 33.2\% of those receiving grommets) had their first grommet insertion at the primary cleft palate and/or lip repair, and 1,004 (12.1\% of all children and $33.3 \%$ of those receiving grommets) underwent multiple grommet insertion procedures (range 2-7) within the first five years of life (Table 1). A total of 167 NHS Trusts across England carried out the grommet insertions.

The rate of grommet insertion before the age of five years declined over the study period $(\mathrm{p}<0.001 ;$ Figure 1). This trend was also observed in the proportion of children having grommets at primary cleft repair and the proportion having multiple grommet procedures.

The multiple grommet insertion rate was higher among those receiving grommets at primary repair compared to those receiving their first grommets at another time $(36.6 \%$ vs. $31.7 \%$. $\mathrm{p}=0.007)$.

The median age for the first grommet insertion was 20 months, and the peak age range for the first procedure was six to 12 months, which is also the peak age of primary palate repair (Fitzsimons et al., 2012). By the age of one year, 12.0\% of all children with CL/P (33.0\% of those receiving grommets) had received grommets. The proportion of children receiving their 
first grommet(s) decreased with each increasing year of age, with just $3.9 \%$ of children with $\mathrm{CL} / \mathrm{P}$ receiving their first grommet(s) at four years of age.

Cleft children with additional anomalies

There were 1,819 (22.0\%) children with additional anomalies (Table 1). Of these, $45.5 \%$ underwent grommet insertion before the age of five years and $27.9 \%$ before the age of one year. There was no significant difference in the proportion of children receiving grommets at primary cleft repair or the proportion receiving multiple grommet insertions between children with and those without additional anomalies.

\section{Cleft type classification}

Increasing cleft type severity $(\mathrm{CL}<\mathrm{CP}<\mathrm{CLP})$ was associated with an increase in both the proportion of children who had grommet insertion before the age of five years $(\mathrm{p}<0.001)$ and of those who had multiple grommet insertions ( $\mathrm{p}=0.007)$ (Table 1 and Figure 2). This trend was also observed in children with additional anomalies (Table 1 and Figure 2).

Of the children with CP, UCLP and BCLP receiving grommets, approximately one third $(30.9 \%-36.2 \%)$ underwent their first grommet insertion at primary cleft repair, compared to only $11.2 \%$ of CL children. CL children were also more likely to be older at the time of their first grommet insertion procedure compared to children with $\mathrm{CP} \pm \mathrm{L}$, with the procedure most frequently occurring at ages three and four years (median age 36.6 months vs. 19.7 months) (Figure 2). 
Socio-economic deprivation

IMD scores were available for 8,109 (98.1\%) children. Affluence was associated with a significantly higher rate of grommet insertion before the age of five years $(p<0.001)$ and in the rate of multiple grommet procedures $(\mathrm{p}=0.039)$ (Table 2$)$.

No significant differences existed between IMD quintile groups in the proportion of children receiving grommets at the time of primary cleft repair or the age at first grommet insertion.

\section{Regional variation}

The regional cleft network in which the child resided was reported for 7,761 (97.7\%) children. The number of NHS Trusts performing grommet insertions varied across the nine regional cleft networks in England, ranging from 12 in the North East to 46 in the South East. The proportion of children receiving grommets before the age of five years ranged from $29.7 \%$ in London to $45.5 \%$ in the North East ( $\mathrm{p}<0.001)$ (Figure 3). A large regional variation was observed in the proportion of children who received their grommets at primary cleft repair $(\mathrm{p}<0.001)$, ranging from $4.4 \%$ (11.6\% of those receiving grommets) in the Yorkshire and Humber region to $24.1 \%$ (58.0\% of those receiving grommets) in the North West.

\section{Background rate of grommet insertions}

A total of 110,785 children, born between 1997 and 2005, received grommets in the first five years of life in England. This corresponds to a grommet insertion rate of $2.1 \%$. Of those receiving grommets, $13.1 \%$ underwent multiple grommet insertions. When excluding children with a cleft, these rates are reduced to $2.0 \%$ and $12.5 \%$, respectively. 


\section{DISCUSSION}

\section{Key findings}

This study found that approximately one in every two children $(45.5 \%)$ with a cleft affecting the palate $(\mathrm{CP} \pm \mathrm{L})$ and one in every 21 children $(4.8 \%)$ with a cleft affecting only the lip received grommets before the age of five years. For comparison, the background rate of grommet insertion among all children (excluding children with a cleft but including those with other medical diagnoses) in England across the same time period (1997-2005 births) was calculated. Approximately one in every 50 children (2.0\%) received grommets in the first five years of life. Of those receiving grommets, one in every eight (12.5\%) underwent multiple grommet insertions compared to one in every three (33.3\%) with a $\mathrm{CP}$ and approximately one in every four $(22.5 \%)$ with a CL before the age of five.

Although this study found a high rate of grommet insertions among children with $\mathrm{CP} \pm \mathrm{L}$, much higher rates have been reported elsewhere. A US study of 86 children with $\mathrm{CP} \pm \mathrm{L}$, born between 2000 and 2005, reported a grommet insertion rate of $98 \%$ and a multiple grommet insertion rate among those receiving grommets of $62 \%$ before the age of five years (Szabo et al., 2010). Their indication for grommet insertion was persistent OME with conductive hearing impairment. Lower thresholds for treatment and six-monthly reviews may explain the higher rates compared to those found in the present study.

In Hong Kong, a study of 84 Chinese children with $\mathrm{CP} \pm \mathrm{L}$ reported a grommet insertion rate of $46 \%$ before the age of two years (Kwan et al., 2011). The comparative figure for the present study is approximately $30 \%$ (grommet insertion rate before the age of two years). During the Hong Kong study, there was no standard protocol for the management of OME among $\mathrm{CP} \pm \mathrm{L}$ patients. Although the incidence of $\mathrm{OME}$ in the first two years was $76.1 \%$, only $16.9 \%$ of children had moderate hearing loss, indicating that many of the grommet insertions 
were likely performed on a prophylactic basis, especially as $80.5 \%$ of the insertions were performed at the time of cleft palate repair. This compares to $45.5 \%$ of the insertions among $\mathrm{CP} \pm \mathrm{L}$ patients in the present study who received grommets in the first two years of life. While we are unable to comment on the indication for grommets in our study, we observed a decline in the rate of grommet insertion and in the proportion of children receiving grommets at primary cleft repair over the study period. This may reflect a move away from the practice of prophylactic grommet insertion and could explain the comparatively low rate of grommet insertion in England.

A lower grommet insertion rate of $38 \%$ was reported in Japan among 108 children with CP who received grommets between the age of one and five years (Kobayashi et al., 2012). Grommets were inserted if OME was diagnosed at one year or later. The multiple insertion rate among those receiving grommets was $35.4 \%$. This is consistent with a study in Korea of 213 children with CP who received grommets during palatoplasty between 1993 and 2006 (Ahn et al., 2012). Thirty-four percent of the children received repeat grommets for recurring chronic OME during a mean follow-up of 4.9 years. We found a similar proportion of children with CP (34.4\%) underwent multiple grommet insertions among those who received their first grommet insertion at the time of primary cleft repair. Interestingly, this was significantly higher than the $30.3 \%$ of those with $\mathrm{CP}$ receiving their first grommets at another time.

In children with CL, we observed a grommet insertion rate that was twice as high as the background rate, even in the absence of additional medical anomalies. The multiple grommet insertion rate of $23 \%$ was also almost twice as high as the background rate. Only a few previous cleft studies have included children with CL when examining grommet insertion rates (Sheahan et al., 2003; Kwan et al., 2011; Kobayashi et al., 2012). In these studies the number of children with CL ranged from seven to 62 . No grommets were inserted in children 
with CL in the studies from Japan (Kobayashi et al., 2012) and Hong Kong (Kwan et al., 2011). The Irish study with 62 patients (age range five months to 27 years, median age seven years) found a grommet insertion rate of 3\% (Sheahan et al., 2003). One possible explanation for the comparatively higher rates of grommet insertions observed in our study could be that hearing problems due to OME are more likely to be identified in children with CL because of the extra monitoring provided as part of their cleft care, which would not be routinely available to the general paediatric population.

The observed increased rate of grommet insertions among children with additional anomalies and syndromes, compared to those with a cleft alone, could reflect an increased incidence of OME. Another possible explanation is that syndromic children may undergo more medical procedures under general anaesthetic, which present an opportunistic time to insert grommets.

We found that the rate of grommet insertion before the age of five years and the rate of multiple grommet insertions was significantly lower for children living in the most deprived areas compared to the most affluent. A link between increased deprivation and lower grommet insertion rates in the general population has been observed previously in Scotland (Bisset and Russell, 1994). Our study may represent the first time these socio-economic disparities have been seen in the cleft population. It is possible that hospitals with more deprived catchment areas take a more conservative approach when treating children with OME. Another possible explanation may be parental expectation, with more affluent parents having a lower threshold for seeking medical attention for perceived fluctuations in hearing (Black, 1985).

Wide variation in the rate of grommet insertion clearly existed over the study period and between the different regional cleft networks within England, suggesting a lack of consensus 
amongst clinicians. Rates may be determined by whether a routine prophylactic approach is taken, with grommets inserted at the time of primary palate repair, or whether a more conservative approach is favoured, whereby grommets are inserted only when signs and symptoms of OME are persistent, as recommended by the NICE guidance (2008). The centralisation of cleft services in England, which was initiated in 1998 and completed by 2007 (Fitzsimons et al., 2012), may have also influenced the declining rate of grommet insertions we observed over the study period.

\section{Strengths and limitations}

To our knowledge, this is the largest study of grommets surgery among children with an orofacial cleft. The study has an important strength: it is based on a national database that aims to include all children treated for a cleft in England since 1997. The HES database contains records of every procedure performed during a particular hospital episode, allowing us to identify all grommet insertion procedures performed on cleft patients. Furthermore, we have shown a high level of agreement between the cleft diagnoses recorded in HES and those submitted by staff directly involved in cleft care to the CRANE database (CRANE Project team on behalf of the Cleft Development Group, 2012), which suggests that the HES database is a reliable data source for cleft care research.

A further strength of this study is that we were able to use the same database to calculate the national background rate of grommets insertion among all live-born children in England across the same time period, which puts into context the grommet insertion rates among children with an orofacial cleft. 
The inclusion of children with CL is rare compared to other studies examining OME and grommet insertion in cleft patients, which have included only children with $\mathrm{CP} \pm \mathrm{L}$ or very few patients with CL alone.

Finally, the inclusion and separate analyses of children with a cleft and additional anomalies is unique. These children account for almost one quarter of all patients with a cleft and our findings show that they have an even higher rate of grommet insertions and re-insertions than those without additional anomalies.

This study has limitations. First, the data we could analyse represent practice observed between 1997 up to 2010 and our findings may therefore not fully reflect grommet surgery today. We observed a declining trend in the rate of grommet insertion over the study period. The publication of the NICE guideline on the surgical management of OME in children in 2008 may have accelerated this trend, although it should be noted that there was no statistical change in the rate of grommet insertions at a national level in the general population in the two years following the publication (Al-Hussaini et al., 2012).

Second, we did not have information on the indication for grommet insertion or audiometric data. Consequently, we do not know what proportion of grommet insertions at the time of primary palate repair was performed as prophylaxis and what proportion was inserted for persistent OME. This information could have helped to explain some of the differences we observed between groups. Similarly, we were unable to examine the outcomes of grommet insertion on long-term hearing, speech development and ear disease (not limited to OME), other than to observe that $33.3 \%$ of children receiving grommets had multiple grommet insertions. 


\section{CONCLUSION}

This study demonstrates that OME places a significant burden on the cleft population and health service in England. Children are more likely to receive grommets if they have a cleft affecting the palate, a more complex cleft type, additional anomalies or syndromes, or if they live in more affluent areas. In addition, we found considerable regional variation. This information is important for the planning and commissioning of cleft services and will also be useful when counselling families of children with a cleft. The impact of socio-economic deprivation on access to grommet surgery should be explored further. We recommend that future research includes outcomes related to the insertion of grommets in order to help identify grommet surgery practice that is of most benefit to children with an orofacial cleft. 


\section{REFERENCES}

Ahn JH, Kang WS, Kim JH, Koh KS, Yoon TH. Clinical manifestation and risk factors of children with cleft palate receiving repeated ventilating tube insertions for treatment of recurrent otitis media with effusion. Acta Oto-laryngologica, 2012; 132(7):702-707. http://informahealthcare.com/doi/abs/10.3109/00016489.2011.652309

Al-Hussaini A, Owens D, Tomkinson A. Have two UK national guidelines had any effect on grommets day-case utilisation and rate over the last 10 years? European Archives of OtoRhino-Laryngology, 2012; 269(9):2053-2056. http://dx.doi.org/10.1007/s00405-011-1860-z

Atkinson M. Surgical management of otitis media with effusion in children - NICE guideline: what paediatricians need to know. Archives of disease in childhood - Education \& practice edition, 2009; 94(4):115-117. http://ep.bmj.com/content/94/4/115.short

Bisset AF, Russell D. Grommets, tonsillectomies, and deprivation in Scotland. BMJ, 1994; 308(6937):1129-1132

Black N. The 'Health Culture' of Families as an Influence on the Use of Surgery for Glue Ear: A Case-Control Study. International Journal of Epidemiology, 1985; 14(4):594-599. http://ije.oxfordjournals.org/content/14/4/594.abstract

CRANE Project team on behalf of the Cleft Development Group. CRANE Database Annual Report 2012. London: Clinical Effectiveness Unit, The Royal College of Surgeons of England 2012.

Cuzick J. A Wilcoxon-type test for trend. Statistics in Medicine, 1985; 4:87-90

Fitzsimons K, Mukarram S, Copley L, Deacon S, van der Meulen J. Centralisation of services for children with cleft lip or palate in England: a study of hospital episode statistics. $B M C$ 
Health Services Research, 2012; 12(1):148. http://www.biomedcentral.com/1472$\underline{6963 / 12 / 148}$

Flynn T, Möller C, Jönsson R, Lohmander A. The high prevalence of otitis media with effusion in children with cleft lip and palate as compared to children without clefts. Int $J$ Pediatr Otorhinolaryngol, 2009; 73(10):1441-1446.

http://www.sciencedirect.com/science/article/pii/S0165587609003863

Gould HJ. Hearing loss and cleft palate: the perspective of time. The Cleft Palate Journal, $1990 ; 27(1): 36-39$

Hospital Episode Statistics. (2010). HES user guide. from www.hscic.gov.uk/hes

Kaplan EL, Meier P. Nonparametric estimation from incomplete observations. Journal of the American Statistical Association, 1958; 53(282):457-481

Kobayashi H, Sakuma T, Yamada N, Suzaki H. Clinical outcomes of ventilation tube placement in children with cleft palate. Int J Pediatr Otorhinolaryngol, 2012; 76(5):718-721. http://dx.doi.org/10.1016/j.ijporl.2012.02.027

Kuo C-L, Tsao Y-H, Cheng H-M, Lien C-F, Hsu C-H, Huang C-Y, Shiao A-S. Grommets for Otitis Media With Effusion in Children With Cleft Palate: A Systematic Review. Pediatrics, 2014; 134(5):983-994. http://pediatrics.aappublications.org/content/134/5/983.abstract

Kwan WMY, Abdullah VJ, Liu K, van Hasselt CA, Tong MCF. Otitis media with effusion and hearing loss in Chinese children with cleft lip and palate. The Cleft Palate-Craniofacial Journal, 2011; 48(6):684-689. http://dx.doi.org/10.1597/10-006

Møller P. Hearing, middle ear pressure and otopathology in a cleft palate population. Acta Oto-laryngologica, 1981; 92(5-6):521-528 
National Collaborating Centre for Women's and Children's Health. Surgical management of otitis media with effusion in children. London: RCOG Press.2008.

National Institute for Health and Clinical Excellence. Surgical management of otitis media with effusion in children. London: NICE.2008.

Office for National Statistics. (2008). Birth statistics, England and Wales (Series FM1). Newport: National Statistics Retrieved from http://www.ons.gov.uk/ons/rel/vsob1/birthstatistics--england-and-wales--series-fm1-/index.html.

Office of the Deputy Prime Minister. Index of Multiple Deprivation, 2004.2004.

Ponduri S, Bradley R, Ellis PE, Brookes ST, Sandy JR, Ness AR. The management of otitis media with early routine insertion of grommets in children with cleft palate -- a systematic review. The Cleft Palate-Craniofacial Journal, 2009; 46(1):30-38

Sheahan P, Miller I, Sheahan JN, Earley MJ, Blayney AW. Incidence and outcome of middle ear disease in cleft lip and/or cleft palate. Int J Pediatr Otorhinolaryngol, 2003; 67(7):785793. http://www.sciencedirect.com/science/article/pii/S0165587603000983

Szabo C, Langevin K, Schoem S, Mabry K. Treatment of persistent middle ear effusion in cleft palate patients. Int J Pediatr Otorhinolaryngol, 2010; 74(8):874-877. http://www.sciencedirect.com/science/article/pii/S0165587610002077

Welsh Government. (2015). StatsWales: Live births by year and age of mother Retrieved from https://statswales.wales.gov.uk/Catalogue/Health-and-Social-Care/Births-Deaths-andConceptions/Births/livebirths-by-year-ageofmother

\section{COMPETING INTEREST}

None declared. 
Table 1. Number (\%) of grommet insertions among children with a cleft before the age of five years, according to cleft type and the absence or presence of additional medical anomalies, year of birth 1997-2005

\section{Number of grommet insertions}

\begin{tabular}{|c|c|c|c|c|c|}
\hline \multirow[b]{2}{*}{ Cleft type } & \multirow[b]{2}{*}{$\mathbf{N}$} & \multicolumn{4}{|c|}{$\mathrm{N}(\%)$} \\
\hline & & 0 & $>0$ & $>$ & 1 \\
\hline \multicolumn{6}{|c|}{ Children with a cleft alone } \\
\hline CL & 1702 & $1630(95.8)$ & $72(4.2)$ & 13 & $(0.8)$ \\
\hline $\mathrm{CP}$ & 2711 & $1575(58.1)$ & 1136 (41.9) & 340 & $(12.5)$ \\
\hline UCLP & 1371 & $745(54.3)$ & $626(45.7)$ & 230 & $(16.8)$ \\
\hline BCLP & 666 & $313(47.0)$ & $353(53.0)$ & 129 & $(19.4)$ \\
\hline All & 6450 & $4263(66.1)$ & $2187(33.9)$ & 712 & $(11.0)$ \\
\hline
\end{tabular}

Children with syndromes or additional anomalies

$\begin{array}{rrrrrrl}\text { CL } & 141 & 124(87.9) & 17(12.1) & 7 & (5.0) \\ \text { CP } & 1382 & 727(52.6) & 655(47.4) & 229 & (16.6) \\ \text { UCLP } & 161 & 75(46.6) & 86(53.4) & 31 & (19.3) \\ \text { BCLP } & 135 & 65(48.1) & 70(51.9) & 25 & (18.5) \\ \text { All } & 1819 & 991(54.5) & 828(45.5) & 292 & (16.1)\end{array}$

All children with a cleft

$\begin{array}{rrrrrr}\text { CL } & 1843 & 1754(95.2) & 89(4.8) & 20 & (1.1) \\ \text { CP } & 4093 & 2302(56.2) & 1791(43.8) & 569 & (13.9) \\ \text { UCLP } & 1532 & 820(53.5) & 712(46.5) & 261 & (17.0) \\ \text { BCLP } & 801 & 378(47.2) & 423(52.8) & 154 & (19.2) \\ \text { All } & 8269 & 5254(63.5) & 3015(36.5) & 1004 & (12.1)\end{array}$

$\overline{\mathrm{CL}}$, cleft lip only; CP, cleft palate only; UCLP, unilateral cleft lip and palate; BCLP, bilateral cleft lip and palate

Hospital episode statistics, England 1997-2011 
Table 2. Number of grommet insertions among children with a cleft before the age of five, according to relative deprivation of area of residence, year of birth 1997-2005

\section{Number of grommet insertions}

\begin{tabular}{|c|c|c|c|c|}
\hline \multirow{2}{*}{$\begin{array}{l}\text { Relative deprivation } \\
\text { (quintiles of IMD) }^{\text {a }}\end{array}$} & \multirow[b]{2}{*}{$\mathbf{N}$} & \multicolumn{3}{|c|}{$\mathrm{N}(\%)$} \\
\hline & & 0 & $>0$ & $>1$ \\
\hline (Most deprived) $1^{\text {st }}$ & 1,909 & $1,262(66.1)$ & $647(33.9)$ & $207(10.8)$ \\
\hline $2^{\text {nd }}$ & 1,641 & $1,044(63.6)$ & $597(36.4)$ & 176 (10.7) \\
\hline $3^{\text {rd }}$ & 1,495 & $941(62.9)$ & $554(37.1)$ & $191(12.8)$ \\
\hline $4^{\text {th }}$ & 1,463 & $913(62.4)$ & $550(37.6)$ & $200(13.7)$ \\
\hline (Least deprived) $5^{\text {th }}$ & 1,601 & $948(59.2)$ & $653(40.8)$ & $228(14.2)$ \\
\hline All & 8,109 & $5,108(63.0)$ & $3001(37.0)$ & 1002 (12.4) \\
\hline
\end{tabular}

${ }^{\mathrm{a}}$ IMD scores missing for $160(1.9 \%)$ children 
Figure 1. Proportion of children receiving: a) grommets, b) grommets at the time of primary cleft repair, and c) multiple grommet insertions, before the age of five years, according to the year of birth, year of birth 1997-2005

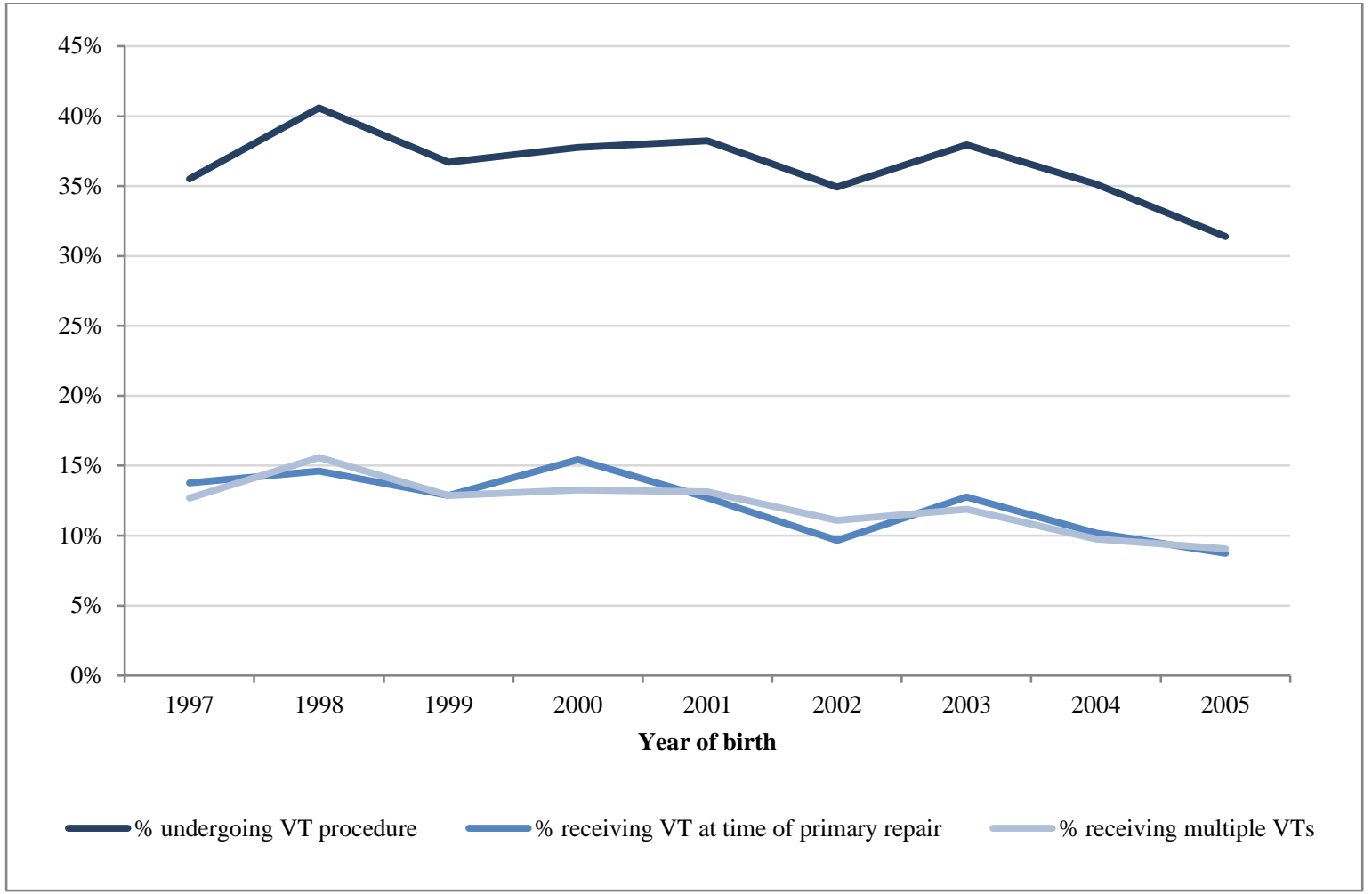

Hospital episode statistics, England 1997-2011 
Figure 2. Kaplan-Meier estimates for time to first grommet insertion procedure within the first five years of life, according to cleft type and the absence or presence of additional anomalies, year of birth 1997-2005

a) Children with a cleft alone

$2 \mathrm{~A}$

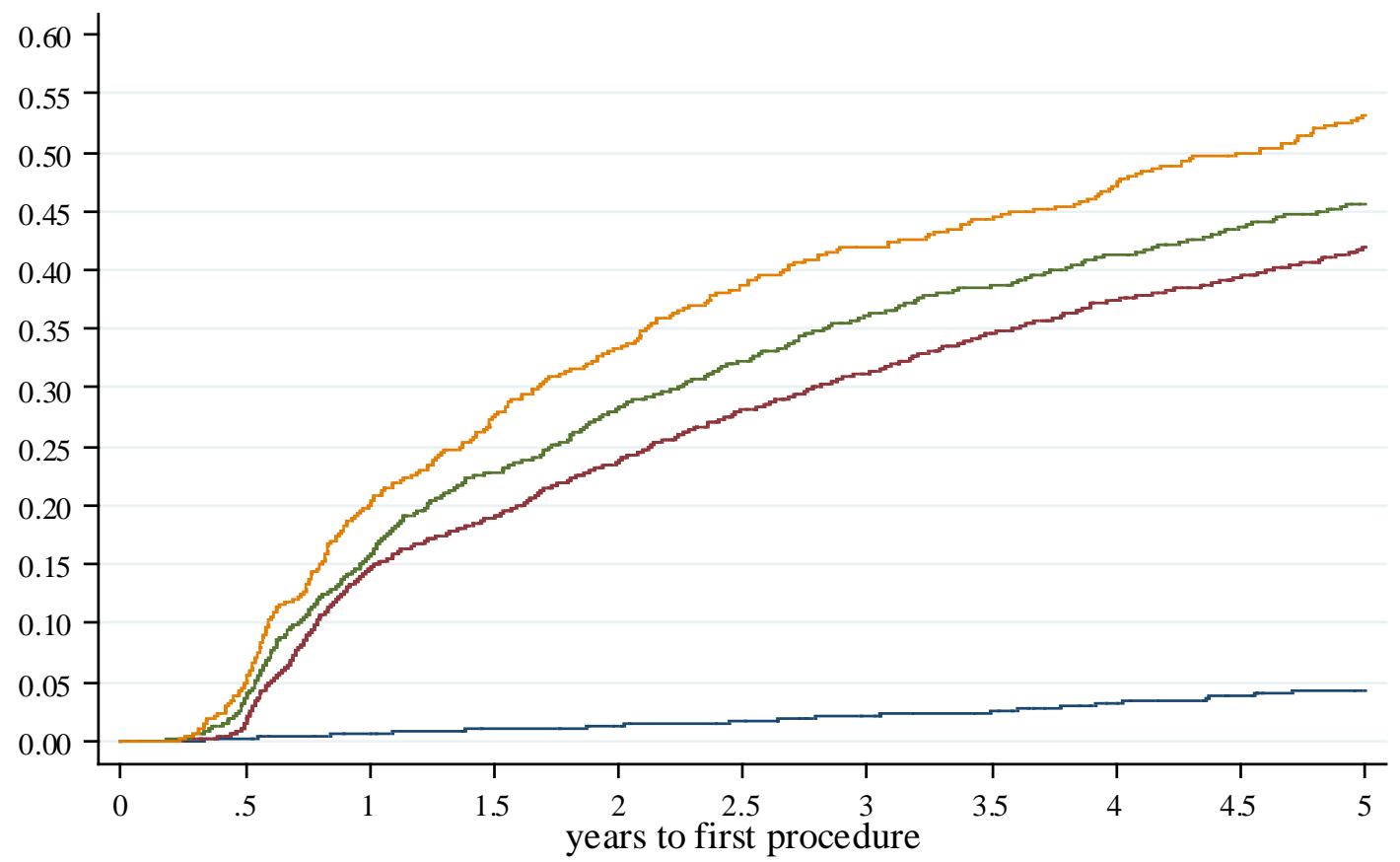

CL

UCLP BCLP 
b) Children with a cleft and additional anomalies

2B

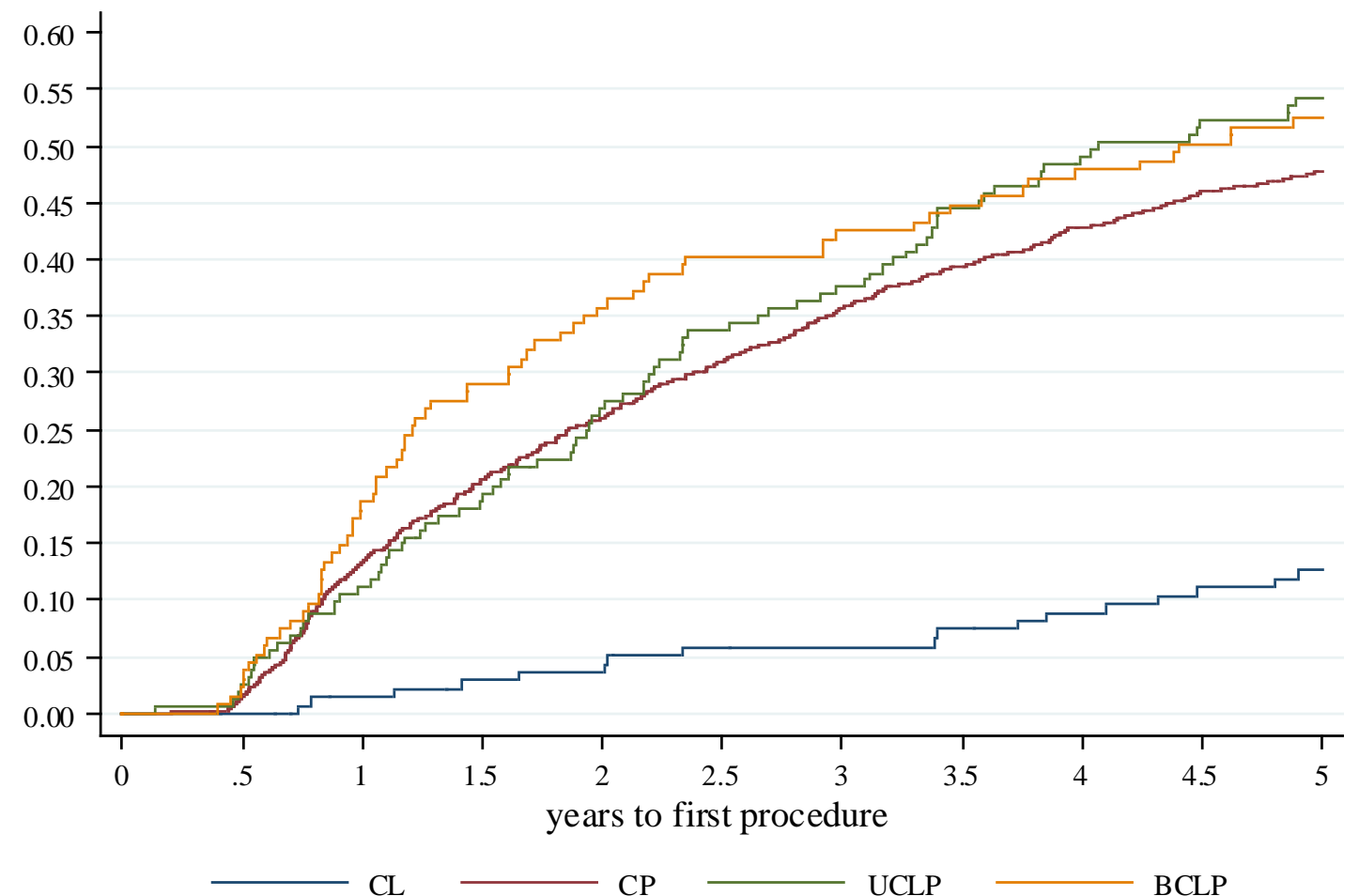

Hospital episode statistics, England 1997-2011 
Figure 3. Proportion of all children with a cleft receiving: a) grommets, b) grommets at the time of primary cleft repair, and c) multiple grommet insertions, before the age of five years, according to the regional cleft network of residence, year of birth 1997-2005

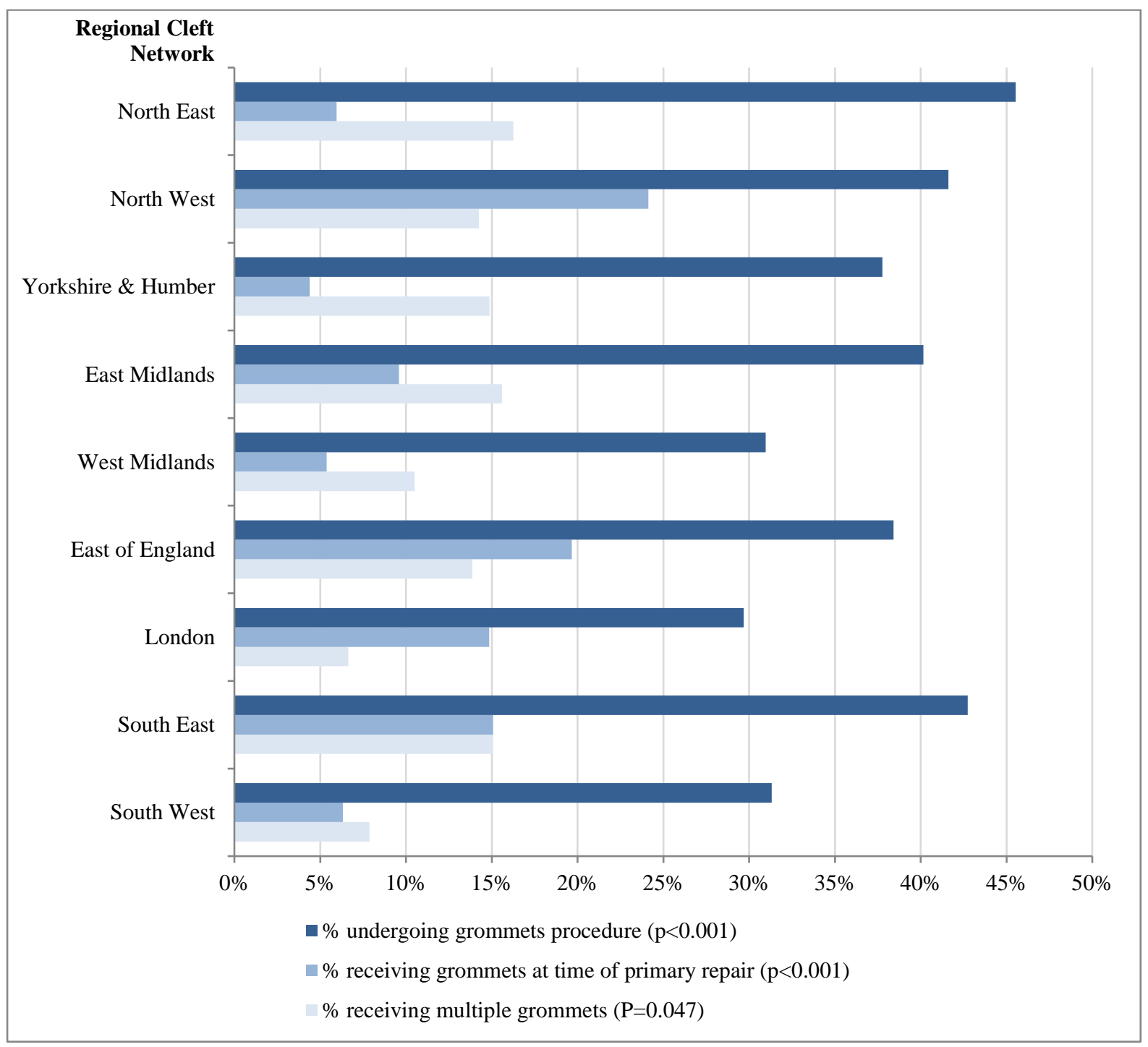

Hospital episode statistics, England 1997-2011; p value comparing proportion across regions 
Appendix 1: Diagnostic codes for syndromes and anomalies used to identify cleft patients with additional anomalies.

Patients were defined as having 'additional anomalies' if there was a record of any of the following codes in any of the fourteen diagnosis code fields for any of that patient's HES episodes. i.e. the diagnosis mention is not necessarily associated with a record containing the patient's first cleft diagnosis/repair.

\begin{tabular}{|l|l|}
\hline Code & Description \\
\hline D821 & Di George's syndrome \\
\hline & Congenital malformations of the nervous system (Q00-Q07) \\
\hline Q00 & Anencephaly and similar malformations \\
\hline Q01 & Encephalocele \\
\hline Q02 & Microcephaly \\
\hline Q03 & Congenital hydrocephalus \\
\hline Q04 & Other congenital malformations of brain \\
\hline Q05 & Spina bifida \\
\hline Q07 & Other congenital malformations of spinal cord \\
\hline & Other congenital malformations of nervous system \\
\hline Q16 & Congenital malformations of ear causing impairment of hearing \\
\hline Q18 & Other congenital malformations of face and neck \\
\hline & \\
\hline & Congenital malformations of the circulatory system (Q20-Q28) \\
\hline Q20 & Congenital malformations of cardiac chambers and connections \\
\hline Q21 & Congenital malformations of cardiac septa \\
\hline Q22 & Congenital malformations of pulmonary and tricuspid valves \\
\hline Q23 & Congenital malformations of aortic and mitral valves \\
\hline Q24 & Other congenital malformations of heart \\
\hline Q25 & Congenital malformations of great arteries \\
\hline Q26 & Congenital malformations of great veins \\
\hline Q27 & Other congenital malformations of peripheral vascular system \\
\hline Q28 & Other congenital malformations of circulatory system \\
\hline & Congenital malformations of lips, not elsewhere classified \\
\hline Q380 & Other congenital malformations of skull and face bones \\
\hline Q75 & $\begin{array}{l}\text { Congenital malformation syndromes due to known exogenous causes, not } \\
\text { elsewhere classified }\end{array}$ \\
\hline Q86 & Other specified congenital malformation syndromes affecting multiple \\
\hline Q87 & \\
\hline & \\
\hline
\end{tabular}




\begin{tabular}{|l|l|}
\hline & Chromosomal abnormalities, not elsewhere classified (Q90-99) \\
\hline Q90 & Down's syndrome \\
\hline Q91 & Edwards' syndrome and Patau's syndrome \\
\hline Q92 & $\begin{array}{l}\text { Other trisomies and partial trisomies of the autosomes, not elsewhere } \\
\text { classified }\end{array}$ \\
\hline Q93 & Monosomies and deletions from the autosomes, not elsewhere classified \\
\hline Q95 & Balanced rearrangements and structural markers, not elsewhere classified \\
\hline Q96 & Turner's syndrome \\
\hline Q97 & $\begin{array}{l}\text { Other sex chromosome abnormalities, female phenotype, not elsewhere } \\
\text { classified }\end{array}$ \\
\hline Q98 & $\begin{array}{l}\text { Other sex chromosome abnormalities, male phenotype, not elsewhere } \\
\text { classified }\end{array}$ \\
\hline Q99 & Other chromosome abnormalities, not elsewhere classified \\
\hline
\end{tabular}

